\title{
Enhanced Expression of Serum miR-410-3p Reflects The Disease Progression in Anti-gp210 Positive Primary Biliary Cholangitis
}

\section{Tiantian Xu}

The Affiliated Hospital of Qingdao University

\section{Pan Wang}

The Affiliated Hospital of Qingdao University

\section{Zhan Wang}

The Affiliated Hospital of Qingdao University

\section{Lin Wang}

The Affiliated Hospital of Qingdao University

\section{Wenshuai Li}

The Affiliated Hospital of Qingdao University

\section{Guirong Sun}

The Affiliated Hospital of Qingdao University

\section{Mingjun Liu ( $\boldsymbol{D}$ jocklmj@hotmail.com )}

The Affiliated Hospital of Qingdao University https://orcid.org/0000-0002-0437-4937

\section{Research Article}

Keywords: Primary biliary cholangitis, anti-gp210, miR-410-3p, miRNAs

Posted Date: November 3rd, 2021

DOl: https://doi.org/10.21203/rs.3.rs-982565/v1

License: (9) This work is licensed under a Creative Commons Attribution 4.0 International License. Read Full License 


\section{Abstract}

Background \& Aims: Anti-gp210 antibodies are highly specific for primary biliary cholangitis (PBC) and are detected in $20 \%-40 \%$ of PBC patients. PBC patients with anti-gp210 positive appear to have more advanced bile duct damage and worst prognosis. Recent studies suggest that miRNAs play a critical role in the progression of PBC. The aim of this study was to explore the relationship between the expression dysregulation of miRNAs in serum from patients with anti-gp210 positive PBC and the disease progression.

Methods: The differentially expressed miRNAs were identified by deep sequencing. The differentially expressed miRNAs were validated by qRT-PCR in PBC patients along with healthy controls and chronic hepatitis $B$ patients. The target genes of differentially expressed miRNAs were predicted using TargetScan and miRDB database. Gene ontology and KEGG pathway analyses were conducted to explore the target genes function.

Results: The expression levels of 60 miRNAs were found to differ significantly between PBC patients with anti-gp210 positive and anti-gp210 negative. MiR-410-3p was validated as the significantly differentially expressed miRNA. MiR-410-3p had a higher level in patients with anti-gp210 positive PBC and was associated with the disease progression. Bioinformatics showed that underlying target genes of miR-410$3 p$ was involved in cell communication, signal transduction, cell apoptosis, and so on. Through KEGG pathway analysis, we suspected that Th1, Th2, Th17 cell differentiation might be associated with the pathogenesis of PBC.

Conclusion: The over-expression of serum miR-410-3p might reflect the disease progression in patients with anti-gp210 positive PBC.

\section{Key Message}

1. The serum expression levels of 60 miRNAs were found to differ significantly between the patients with anti-gp210 positive and anti-gp210 negative PBC.

2. Serum miR-410-3p had a higher level in patients with anti-gp210 positive PBC in comparison to patients with anti-gp210 negative PBC and healthy controls.

3. miR-410-3p is associated with the disease progression and thus inhibiting the expression of miR410-3p could become a potential molecular therapeutic target.

4. Target genes and enriched pathways highlight the importance of T, B cell differentiation and activation in the disease progression of PBC.

\section{Introduction}

Primary biliary cholangitis (PBC) is an autoimmune liver disease pathologically characterized by destruction of middle and small intrahepatic bile ducts and progressive cholestasis, resulting in periportal 
inflammation, fibrosis and cirrhosis[1]. PBC mainly has an influence on mostly middle-aged women[2]. Etiologically, PBC is currently believed to be a combination of susceptibility genes, environmental risk factors and immune disorders[3]; however, the exact pathogenesis remains unclear. The highly sensitive and specific diagnostic hallmark of PBC is the presence of anti-mitochondrial antibody (AMA) as $90 \%-95 \%$ patients with PBC are AMA positive $[4,5]$. In addition, the presence of a number of anti-nuclear antibodies (ANA) has been reported in PBC patients $[4,6]$. Among these ANA, antibodies to the nuclear envelope glycoprotein 210 (gp210) are highly specific for PBC and are detected in $20 \%-40 \%$ of PBC patients[7]. Affected patients with anti-gp210 positive PBC appear to have more advanced bile duct damage and worst prognosis than the patients with anti-gp210 negative PBC for reasons poorly understood[8]. Moreover, the degree of this anti-gp210 expression positively is associated histologically with the disease activity, including portal inflammation, interface hepatitis and lobular inflammation[9].

MicroRNAs (miRNAs) are a group of endogenous non-coding small RNA molecules of 21-23 nucleotides in length which bind imperfectly to the complementary sequences in the $3^{\prime}$ untranslated region (UTR) of target messenger RNAs and repress their transcription and translation[10]. MiRNAs are involved in several important biological processes including cell division, differentiation, proliferation, apoptosis, carcinogenesis, and immune function, and so on[11]. Furthermore, evidence is growing that miRNAs expression profiles are different among different liver diseases and some miRNAs may relate to the progression of liver diseases $[12,13]$. Similar to other autoimmune diseases, recently studies concentrated on the specific miRNAs expression profiles in either tissues or serum of PBC patients. For example, the expression of miRNA-506 is upregulated in bile duct epithelial cells of PBC patients which is related to decreased AE2 and InsP3R3 activity[14]. MiRNAs have been shown to be altered in the serum of PBC patients compared with healthy controls or patients with chronic viral hepatitis[15], implicating them as possible non-invasive diagnostic biomarkers for $\mathrm{PBC}$ and potential players in the disease pathogenesis.

Hitherto, the knowledge on serum miRNAs in PBC is limited and a broad profiling of serum miRNAs is lacking. In the present study, we evaluated patterns of miRNAs expression in serum samples from patients with anti-gp210 positive and anti-gp210 negative PBC using the comprehensive deep sequencing technique. Furthermore, quantitative real-time PCR (qRT-PCR) was used to further verify the significantly differentially expressed miRNAs, and the difference of miRNAs expression levels in serum between patients with anti-gp210 positive and anti-gp210 negative PBC was compared to monitor the disease progression of PBC at the molecular level. In addition, computer analysis of differentially expressed miRNAs was conducted to predict the downstream target genes and their biological functions.

\section{Materials And Methods}

\subsection{Patients and controls}

The study involved 82 PBC patients recruited from The Affiliated Hospital of Qingdao University between December 2020 and July 2021, including 36 patients with anti-gp210 positive PBC and 46 patients with 
anti-gp210 negative PBC. All patients were diagnosed according to the EASL Practice Guideline[16]. Exclusion criteria included individuals infected with chronic viral hepatitis, patients presented with rheumatoid arthritis, osteoarthritis, autoimmune hepatitis, overlapping syndrome, and other malignancies. Liver cirrhosis was diagnosed on the basis of clinical/biochemical and imaging features. Twenty patients underwent liver biopsy. Twenty patients with chronic hepatitis $B(\mathrm{CHB})$ and 20 healthy controls $(\mathrm{HC})$ were also recruited in this study. Detailed clinical data on included patients and controls are summarized in Table 1 and Table 2. The experimental protocol was approved by the Ethics Committee of The Affiliated Hospital of Qingdao University. An informed written consent was obtained from each individual.

Based on natural course staging, PBC progression was divided into four phases. Phase I is characterized by positive AMA and normal liver function. Phase II is characterized by abnormal liver function without clinical symptoms. Phase III is characterized by fatigue and pruritus. Phase IV is characterized by upper gastrointestinal bleeding, ascites, hepatic encephalopathy, jaundice[17]. Cholangitis activity was graded as CA0-3. Response to PBC treatment was defined by a decrease in alkaline phosphatase (ALP) of greater than $40 \%$ of the baseline value or normal level after one year of treatment with ursodeoxycholic acid (UDCA), according to the Barcelona criteria[17, 18]. All of 82 PBC patients included 62 patients with UDCA treatment for more than one year, 10 patients with less than one year of UDCA treatment, and 10 patients initially diagnosed without treatment with UDCA.

\subsection{Serum collection}

Serum was isolated from venous blood samples from all subjects by centrifugation at $4500 \mathrm{~g}$ for 10 minute.

\subsection{Deep Sequencing Using an Illumina Sequencer}

A deep sequencer is able to analyze a large number of small-molecule RNA species in a single run, making it an ideal tool for this type of study. The instrument employed for this purpose was an Illumina NextSeq 500 (Illumina, CA, USA). After exposure, total RNA was extracted from $250 \mu$ l of serum from each of the 3 patients with anti-gp210 positive PBC and 3 patients with anti-gp210 negative PBC and 3 healthy controls using Trizol LS Reagent (Invitrogen, CA, USA). Total RNA was qualified and quantified using a NanoDrop ND2000 and Agilent 2100 bioanalyzer (ThermoFisher Scientific, MA, USA). MiRNA libraries were then constructed from total RNA using the NEB Multiple Small RNA Library Prep Set (Illumina). The sequencing images were analyzed in real time using SCS 2.8 software (Illumina), with a 32-mer singleend sequence. Base calling was performed using RTA 1.8 software.

\subsection{MiRNA sequencing data processing}

The raw miRNA sequence reads were processed using the following steps by removing low-quality sequences, sequences with 5 primer contaminants or poly A, sequences without 3 primer or insertion, and sequences shorter than 18 nucleotides[19]. After filtering, the clean reads were mapped to the reference genomes and miRBase (June 2014 release), allowing one nucleotide base mismatch. The levels of digital 
expression were neutralized by considering the length of the miRNAs. The total number of miRNA reads generated in each library was expressed using trimmed mean of M values (TMM) normalization. Read counts for each isolated miRNA were normalized to the total number of miRNA reads, and then the ratio was multiplied by a constant set to $1 \times 10^{6}$. Hierarchical clustering was performed using the $\mathrm{R}$ platform and a heatmap described as using a function of heatmap. 2 in gplots [20, 21].

\subsection{Quantitative real-time polymerase chain reaction (qRT- PCR)}

In addition to initial samples analyzed by deep sequencing, more serum samples from patients with PBC and $\mathrm{CHB}$ and healthy controls (a total of 82, 20 and 20 samples in each group) were used in a validation study employing qRT-PCR. miRNA extracted from $300 \mu \mathrm{l}$ serum was firstly used to perform adding poly A tailing reaction, and then, cDNA was synthesized using a HG TaqMan miRNA RT Kit (HaiGene, Haerbin, China), following the manufacturer's protocol. qRT-PCR was conducted for detection of the identified miRNAs in 20- $\mu$ PCR reaction volumes using QuantStudio Dx Real-time PCR System (Applied Biosystems, USA) and the HG TaqMan miRNA qPCR Kit (HaiGene, Haerbin, China) according to the manufacturer's instructions. We used the following primers: hsa-miR-410-3p, forward, 5'-

CGCGCGAATATAACACAGATG-3', reverse, 5'-GAGAACAGCTCTGTGTTATAT-3'. The cycles were as follows: initial denaturation at $95^{\circ} \mathrm{C}$ for $15 \mathrm{~min}$, denaturation at $95^{\circ} \mathrm{C}$ for $10 \mathrm{~s}$, annealing at $60^{\circ} \mathrm{C}$ for $60 \mathrm{~s}$ for 40 cycles. Levels of expression of the identified miRNAs detected by qRT-PCR were normalized to hsa-miR16. The relative expression level of each miRNA was calculated using the equation $2^{-\Delta \Delta C t}$.

\subsection{Bioinformatic analysis of differentially expressed miRNAs}

To evaluate functions of candidate miRNAs of PBC patients, target genes prediction were performed using the TargetScan database (http://www.targetscan.org/cgi-bin/targetscan/vert_71/) and miRDB database (http://www.mirdb.org/cgi-bin/). The functional annotation of target genes was performed using the DAVID 6.7 (https://david.ncifcrf.gov/summary.jsp). DAVID provides functional annotation of a gene list based on gene ontology (GO) terms and the Kyoto Encyclopedia of Genes and Genomes (KEGG) pathway databases (http://www.genome.jp/)[1]. Biological processes (BP), cellular component (CC), and molecular function (MF) categories were explored through GO analysis. KEGG analysis is a systematic approach to assess gene function for the discovery of bioregulatory pathways[22].

\subsection{Statistical analysis}

For the discovery study, the non-normally distributed continuous variables of differentially expressed miRNAs between PBC patients and healthy controls, as wells as, between patients with anti-gp210 positive and anti-gp210 negative PBC were compared by Mann-Whitney $U$ test. Different miRNAs were compared using significant analysis and false discovery rate (FDR) analysis. The FDR was calculated to correct the $p$-value. FDR $\leq 0.2$ indicated smaller errors in judging $p$-values. 
For the validation study, Graphpad Prism 6.0 Software or IBM SPSS 22 Software was used for the subsequent analysis in the study. Continuous variables were shown as mean \pm SD (standard deviation) for normal distribution and median with interquartile range (IQR) for non-normal distribution. Categorical variables were presented as the number (or percentage). Two-sided Student's t-test, one-way ANOVA and S-N-K multiple comparison post hoc tests were applied to compare continuous statistics that were normally distributed. Mann-Whitney $\mathrm{U}$ test and Wilcoxon signed rank test, Kruskal-Wallis and Dunn's multiple comparison post hoc tests were applied to compare continuous data that were not normally distributed as indicated. $\chi^{2}$ test was applied to categorical data. Receiver operating characteristic (ROC) analysis was performed to determine the optimal cut-off values of continuous data, and to estimate the sensitivity and specificity. Differences were considered statistically significant at $P<0.05$.

\section{Results}

\subsection{Study population characteristics}

Demographic characteristics and blood biochemical parameters of PBC patients as well as healthy controls, patients with chronic hepatitis B were summarized in Table 1. The characteristics of patients with anti-gp210 positive and anti-gp210 negative PBC were presented in Table 2. We found that patients with anti-gp210 positive PBC had a higher level of some indicators, including ALP, GGT, TBil, compared to patients with anti-gp210 negative PBC; however, the basal level of ALB was lower in the patients with antigp210 positive PBC (Table 2).

\subsection{MiRNAs expression profiles of serum in PBC and healthy controls}

Using single-end deep sequencing, we analyzed circulating miRNAs detected in 9 serum samples from 3 patients with anti-gp210 positive PBC and 3 patients with anti-gp210 negative PBC, and 3 healthy controls. After normalization of the raw data and random variance model, we found 192 miRNAs were differentially expressed in PBC patients compared to the healthy controls. In particular, the analysis identified 118 up-regulated miRNAs ( $>2.0$-fold) and 74 down-regulated miRNAs ( $<0.5$-fold) in PBC patients compared with the healthy controls. Moreover, the expression levels of 60 miRNAs were found to differ significantly between the patients with anti-gp210 positive and anti-gp210 negative PBC. Heat mapping and hierarchical clustering demonstrated that the miRNA expression profiles from patients with antigp210 positive and anti-gp210 negative PBC were clustered differently from healthy controls (Fig. 1). Notably, there was a characteristic difference in the patterns of miRNAs expression between patients with anti-gp210 positive and anti-gp210 negative PBC.

\subsection{Validation of differentially expressed miRNAs by qRT- PCR}

We selected miR-410-3p which had a higher expression level in 3 patients with anti-gp210 positive PBC compared to 3 patients with anti-gp210 negative PBC and 3 healthy controls and was uniformly 
expressed in 3 patients with anti-gp210 positive PBC as an alternative miRNA for further analysis. Validation of miR-410-3p expression was performed in all samples from PBC patients $(n=82)$, healthy controls $(n=20)$ and CHB patients $(n=20)$. The expression of miR-410-3p was significantly up-regulated in PBC patients compared with the healthy controls $(p<0.0001)$ and the CHB patients $(p<0.0001)$ (Fig. 2a). ROC analysis revealed that serum miR-410-3p yielded area under the curve (AUC) values of $0.775(95 \% \mathrm{Cl}$, $0.688 \sim 0.862 ; p<0.0001)$, with a corresponding sensitivity of $64.6 \%$ and a specificity of $95.0 \%$ in discriminating PBC from healthy controls when the optimal critical value was 1.44 (Fig. 2b). Besides, serum miR-410-3p yielded AUC values of $0.791(95 \% \mathrm{Cl}, 0.695 \sim 0.887 ; p<0.0001)$, and when the cut-off value was 1.31 , a sensitivity was $80.5 \%$ and a specificity was $70.0 \%$ in distinguishing PBC from $\mathrm{CHB}$ patients (Fig. 2c).

Expression of miR-410-3p in patients with anti-gp210 positive PBC has shown a 6.9-fold increase compared to healthy controls ( $p<0.0001$; Fig. $3 a$ ) and 8.2 -fold increase compared to patients with antigp210 negative PBC ( $<<0.0001$; Fig. 3a), which was consistent with the deep sequencing results. The serum level of miR-410-3p was higher in cirrhotic group in comparison to non-cirrhotic group in patients with anti-gp210 positive ( $<<0.01$; Fig. $3 b)$ and anti-gp210 negative $(p<0.001$; Fig. $3 b)$ PBC. Furthermore, in patients with anti-gp210 positive PBC, these patients with nature course phase III-IV had a higher level of miR-410-3p compared to patients with nature course phase I-II ( $p<0.001$; Fig. $3 c)$. When miR-410-3p expression was compared in relation to $C A$, the miR-410-3p expression level was substantially enhanced in PBC patients with a CA of 2-3 than in those with a CA of 0-1 ( $<<0.05$; Fig. 3d). In 15 patients with PBC showing non-response to UDCA treatment, the expression of miR-410-3p was significantly increased compared to that in healthy controls $(\mathrm{p}<0.0001$; Fig. 3e) and PBC patients responding to UDCA treatment $(p<0.05$; Fig. 3e)

\subsection{Target genes prediction and GO, KEGG pathway analysis of miR-410-3p}

We predicted the target genes of miR-410-3p using online search algorithms for target genes prediction (TargetScan, http://www.targetscan.org; miRDB, http://www.mirdb.org). There are 539 target genes for miR-410-3p. The potential target genes in relation to differentiation, activation, proliferation, receptor signaling pathways, and homeostasis of T cell or B cell include PPP3CB, ETS1,TNFSF11, TNFSF9, FOXF1 and RUNX1T1, CBFB, SP3 (Fig. 4). Other target genes associated with the occurrence and development of liver fibrosis are VEGFA, SMAD7, COL4A3 and FGFR2, ACVR1C.

For these putative target genes, GO analysis and KEGG pathway analysis were applied to display the miRNA-gene regulatory network. The most significant GO categories for the target genes of miR-410-3p are cell communication, regulation of cellular metabolic process and regulation of signal transduction (Table 3). Besides, these predicted genes were entered in gene set enrichment analysis (GSEA) using KEGG database. KEGG pathways based on potential target genes of miR-410-3p mainly include TGF $\beta$, MAPK, Wnt signaling pathways and Th1, Th2, Th17 cell differentiation, and so on (Table 4). 


\section{Discussion}

MiRNAs, as a new class of regulatory molecules, have recently been investigated as potential critical factors in the disease progression of PBC patients $[1,12]$. Moreover, the fact that circulating miRNAs can reflect the pathogenic states in autoimmunity suggests that these molecules are promising disease biomarkers and potential therapeutic targets[11]. In the present study, we examined the miRNA signature in the serum of PBC patients. To our knowledge, there have been no previous studies investigating a comprehensive miRNA profiling in the serum of patients with anti-gp210 positive and anti-gp210 negative PBC.

We found that patients with anti-gp210 positive PBC had a higher level of some indicators, including ALP, GGT, TBil, compared to patients with anti-gp210 negative PBC; however, the basal level of ALB was lower in the patients with anti-gp210 positive PBC. This suggested that anti-gp210 might be associated with a more severe cholestatic manifestation in PBC patients[8, 9]. In this study, we identified specific patterns of miRNA expression in serum from patients with anti-gp210 positive and anti-gp210 negative PBC using both deep sequencing and qRT-PCR. Hierarchical clustering of the 60 identified miRNA profiles highlighted that the patterns characterizing the patients with anti-gp210 positive PBC differed from those characterizing the patients with anti-gp210 negative PBC, suggesting that some miRNAs may be involved in the production of anti-gp210 antibodies, and even the clinical progression of PBC, or that some cases may progress as a result of specific changes in the expression of some miRNAs. We suspected that these miRNAs might play important roles in the disease process, and we focused on one potentially important miRNA, miR-410-3p, which had previously been reported in autoimmune diseases including rheumatoid arthritis, osteoarthritis and various cancers $[10,23,24]$. We found that expression of miR-410-3p changed significantly: real-time PCR confirmed that miR-410-3p in serum from patients with anti-gp210 antibodies positive PBC showed higher expression in comparison with the patients with anti-gp210 antibodies negative $\mathrm{PBC}$ and healthy controls. Besides, preliminary analysis based on the ROC analysis indicated a high predictive ability of miR-410-3p in distinguishing PBC from CHB patients and healthy controls, implicating it as potential non-invasive diagnostic biomarkers for PBC. However, the high variability of miR-410-3p among individuals or the methodological standardization issues in using miRNAs for clinical diagnosis might limit the utility of miR-410-3p as a diagnostic biomarker. Adequate studies should be conducted to verify the potential of miR-410-3p as an early biomarker for PBC, as well as its prognostic value, also in combination with other clinical indicators.

Furthermore, we demonstrated that miR-410-3p had a higher level in cirrhotic group in contrast to noncirrhotic group in patients with anti-gp210 positive and anti-gp210 negative PBC, suggesting that miR410-3p may be involved in the development of liver cirrhosis. Besides, in patients with anti-gp210 positive PBC, the expression of miR-410-3p was significantly enhanced in advanced clinical course, which implicated us that miR-410-3p might play an important role in the progression of PBC. Noteworthy, the expression level was significantly higher in PBC patients with a CA of 2-3 than in those with a CA of 0-1. In particular, the expression of miR-410-3p in PBC patients showing no response to UDCA treatment was significantly increased compared to that in healthy controls and PBC patients responding to UDCA 
treatment. Previous studies showed that the patients with anti-gp210 positive PBC had more severe cholangitis and poorer response to UDCA treatment in comparison with the patients with anti-gp210 negative PBC $[9,25,26]$. According to these data, it is reasonable to assume that the over-expression of miR-410-3p in patients with anti-gp210 positive PBC may be associated with the cholangitis activity and aggravate bile duct injury. Moreover, inhibition of miR-410-3p expression may be a potential therapeutic target.

It has been reported that miR-410-3p is abnormally expressed in different kinds of diseases, including cancers and autoimmune diseases and regulates multiple biological processes, such as cell proliferation, invasion and migration, apoptosis and differentiation, drug resistance [27-29]. There are studies showing that miR-410-3p is significantly up-regulated in the lung cancer and colorectal cancer, and enhances the proliferation, invasion, and migration of cancer cells [30,31]. In contrast, miR-410-3p was shown to suppress the cell proliferation, invasion, and migration of breast cancer, pancreatic cancer and prostatic cancer $[23,32]$. These data indicate that dysregulation of miR-410-3p occurs in a tissue-specific manner in different types of diseases. One study reported that miR-410-3p can protect chondrocytes from apoptosis by regulating NF-kB signaling pathway via targeting HMGB1 in osteoarthritis[10]. Additionally, previous studies have shown that miR-410-3p suppresses cytokine release from fibroblast-like synoviocytes by regulating NF-kB signaling in rheumatoid arthritis[24]. However, the relationship between miR-410-3p and PBC has not been elucidated yet. Thus, our study is the first to suggest a possible link between miR-410-3p and PBC. Our results suggested that miR-410-3p might play an important proinflammatory role in the pathogenesis of PBC. Also, future in vivo and in vitro mechanistic studies are needed to elucidate the role of miR-410-3p in the pathogenesis of PBC.

In this study, GO analysis was used to obtain insights into the molecular function and biological processes of our identified target genes of miR-410-3p. The analysis highlighted the possible role of miR410-3p involved in the progression of PBC. And, these important biological processes mainly included cell communication, signal transduction, cell proliferation, cell differentiation, cell apoptosis and cellular metabolic process in GO categories. On the other hand, KEGG pathway analysis was performed to further investigate the relationship between the upregulated miR-410-3p and PBC pathogenesis. Several signaling pathways previously proposed to impact $\mathrm{PBC}$ pathogenesis were also identified as candidates in our study including TGF- $\beta$ signaling, Th1 and Th2 cell differentiation, T cell receptor signaling and Th17 cell differentiation $[2,33,34]$, and so on. Again, further studies are needed to explore how the overexpression of miR-410-3p influences the pathogenesis of PBC.

Progression of PBC is largely due to sustained activation of autoantigen-specific B cells in blood and the expansion of autoantigen specific $\mathrm{CD} 4^{+} \mathrm{T}$ cells, $\mathrm{CD} 8^{+} \mathrm{T}$ cells as well as NK and NKT cells in the liver, ultimately leading to bile duct injury[34]. By target genes prediction of miR-410-3p, we observed that the critical target genes are mainly involved in T, B cell proliferation, differentiation and activation, such as ETS1, CBFB and TNFSF9 and so on. It is reasonable to assume that miR-410-3p might facilitate T,B cell differentiation, activation and even autoantibody production by targeting certain genes in PBC. The transcription factor E26 transformation-specific-1 (Ets-1) is a negative regulator of Th17 cell and B cell 
differentiation[35]. Deficiency of Ets-1 promotes Th17 cells differentiation and IL22 and IL23 receptor over-expression. Besides, Ets-1 deficiency can drive terminal differentiation of B cells into immunoglobulin M (IgM) - and immunoglobulin G (IgG) - secreting plasma cells [36]. Previous studies have shown that Ets-1 1Das the target of many miRNAs, such as miR-326, is involved in a variety of autoimmune diseases, including multiple sclerosis (MS), systemic lupus erythematosus (SLE) and idiopathic thrombocytopenic purpura (ITP) $[35,37,38]$. Whether miR-410-3p also promotes the differentiation and activation of Th17 cells and B cells by regulating Ets-1 or other target genes in PBC patients remains to be verified by further in vivo and in vitro experiment.

In conclusion, the circulating miRNAs are significantly altered in serum of PBC patients compared to healthy controls. The miRNAs expression profiles of patients with anti-gp210 positive PBC are obviously different from those of patients with anti-gp210 negative PBC. The over-expression of miR-410-3p in patients with anti-gp210 positive PBC might be specifically associated with the disease progression, and thus inhibiting the expression of miR-410-3p could become a potential molecular therapeutic target. This study provides a novel perspective into the role of miRNAs in PBC pathogenesis; however, the precise mechanism needs further experimental investigation.

\section{Declarations}

\section{Ethics approval and consent to participate}

All procedures performed in studies involving human participants were in accordance with the ethical standards of the institutional and/or national research committee and with the 1964 Helsinki declaration and its later amendments or comparable ethical standards. This article does not contain any studies with animals performed by any of the authors.

\section{Consent for publication}

N/A

\section{Availability of data and materials}

The datasets used and/or analyzed during the current study are available from the corresponding author on reasonable request.

\section{Informed consent}

Informed consent was obtained from all individual participants included in the study.

\section{Conflicts of interest disclosure}

The authors reported no conflict of interests for the work described here.

\section{Funding}


This work was funded by grants from the National Natural Science Foundation of China [81971997] and the Young Scientific Research Fund [The Affiliated Hospital of Qingdao University, 20141201].

\section{Author Contributions}

Conceptualization, Mingjun Liu; data curation, Lin Wang and Wenshuai Li; formal analysis, Tiantian Xu and Zhan Wang; methodology, Mingjun Liu and Guirong Sun; project administration and funding acquisition, Mingjun Liu; resources, Pan Wang; supervision, Tiantian Xu; validation, Tiantian Xu and Pan Wang; writing-original draft, Tiantian Xu; writing-review \& editing, Mingjun Liu and Guirong Sun. All authors read and approved the final manuscript.

\section{Acknowledgments}

$\mathrm{N} / \mathrm{A}$

\section{References}

1. Wang X, Wen X, Zhou J, Qi Y, Wu R, Wang Y, Kui Y, Hua R, Jin Q (2017) MicroRNA-223 and microRNA21 in peripheral blood $B$ cells associated with progression of primary biliary cholangitis patients.PloS one, 12(9),e018424292. https://doi.org/10.1371/journal.pone.0184292

2. Song $Y$, Yang H, Jiang $K$, Wang BM, Lin R (2018) miR-181a regulates Th17 cells distribution via upregulated BCL-2 in primary biliary cholangitis. Int Immunopharmacol 64:386-393. https://doi.org/10.1016/j.intimp.2018.09.027

3. Joshita S, Umemura T, Tanaka E, Ota M (2018) Genetics and epigenetics in the pathogenesis of primary biliary cholangitis. Clin J Gastroenterol 11(1):11-18. https://doi.org/10.1007/s12328-0170799-z

4. Toh BH (2017) Diagnostic autoantibodies for autoimmune liver diseases. Clinical \& translational immunology 6(5):e139. https://doi.org/10.1038/cti.2017.14

5. Laschtowitz A, de Veer RC, Van der Meer AJ, Schramm C (2020) Diagnosis and treatment of primary biliary cholangitis. United European gastroenterology journal 8(6):667-674. https://doi.org/10.1177/2050640620919585

6. Reiberger T, Halilbasic E, Kontic M, Eigenbauer E, Trauner M (2019) Characteristics of AMA-M2(+) and SP100/GP210(+) primary biliary cholangitis (PBC) at diagnosis and their impact on prognosis. Zeitschrift für Gastroenterologie, 2019;57(5): -.https://doi.org/10.1055/s-0039-1691905

7. Honda A, Ikegami T, Matsuzaki Y (2015) Anti-gp210 and anti-centromere antibodies for the prediction of PBC patients with an incomplete biochemical response to UDCA and bezafibrate. Hepatology research: the official journal of the Japan Society of Hepatology 45(8):827-828. https://doi.org/10.1111/hepr.12461

8. Haldar D, Janmohamed A, Plant T, Davidson M, Norman H, Russell E, Serevina O, Chung K, Qamar K, Gunson B et al (2021) Antibodies to gp210 and understanding risk in patients with primary biliary 
cholangitis. Liver international: official journal of the International Association for the Study of the Liver 41(3):535-544. https://doi.org/10.1111/liv.14688

9. Nakamura M (2014) Clinical significance of autoantibodies in primary biliary cirrhosis. Semin Liver Dis 34(3):334-340. https://doi.org/10.1055/s-0034-1383732

10. Pan H, Dai H, Wang L, Lin S, Tao Y, Zheng Y, Jiang R, Fang F, Wu Y (2020) MicroRNA-410-3p modulates chondrocyte apoptosis and inflammation by targeting high mobility group box 1 (HMGB1) in an osteoarthritis mouse model. BMC Musculoskelet Disord 21(1):486. https://doi.org/10.1186/s12891-020-03489-7

11. Zhang L, Wu H, Zhao M, Chang C, Lu Q (2020) Clinical significance of miRNAs in autoimmunity. J Autoimmun 109:102438. https://doi.org/10.1016/j.jaut.2020.102438

12. Katsumi T, Ninomiya M, Nishina T, Mizuno K, Tomita K, Haga H, Okumoto K, Saito T, Shimosegawa T, Ueno $Y(2016)$ MiR-139-5p is associated with inflammatory regulation through c-FOS suppression, and contributes to the progression of primary biliary cholangitis. Lab Invest 96(11):1165-1177. https://doi.org/10.1038/labinvest.2016.95

13. Huang C, Xing X, Xiang X, Fan X, Men R, Ye T, Yang L (2020) MicroRNAs in autoimmune liver diseases: from diagnosis to potential therapeutic targets. Biomedicine \& pharmacotherapy $=$ Biomedecine \& pharmacotherapie 130:110558. https://doi.org/10.1016/j.biopha.2020.110558

14. Rodrigues PM, Perugorria MJ, Santos-Laso A, Bujanda L, Beuers U, Banales JM (2018) Primary biliary cholangitis: A tale of epigenetically-induced secretory failure? J Hepatol 69(6):1371-1383. https://doi.org/10.1016/j.jhep.2018.08.020

15. Wasik U, Kempinska-Podhorodecka A, Bogdanos DP, Milkiewicz P, Milkiewicz M (2020) Enhanced expression of miR-21 and miR-150 is a feature of anti-mitochondrial antibody-negative primary biliary cholangitis. Mol Med 26(1):8. https://doi.org/10.1186/s10020-019-0130-1

16. European Association for the Study of the Liver. Electronic address: easloffice@easloffice.eu, \& European Association for the Study of the Liver (2017) EASL Clinical Practice Guidelines: The diagnosis and management of patients with primary biliary cholangitis. Journal of hepatology, 67(1), 145-172. https://doi.org/10.1016/j.jhep.2017.03.022

17. Al-Harthy N, Kumagi T (2012) Natural history and management of primary biliary cirrhosis. Hepatic medicine: evidence and research 4:61-71. https://doi.org/10.2147/HMER.S25998

18. Örnolfsson KT, Lund SH, Olafsson S, Bergmann OM, Björnsson ES (2019) Biochemical response to ursodeoxycholic acid among PBC patients: a nationwide population-based study. Scand J Gastroenterol 54(5):609-616. https://doi.org/10.1080/00365521.2019.1606931

19. Chen Y, Li D, Deng F, Cao Y, Fu Y, Zhu M, Schultz D, Shekh K, Tang S (2021) Integrated highthroughput small RNA and transcriptome sequencing unveil the shape-dependent toxicity of nanoalumina in rat astrocytes. Environmental Sciences Europe. 2021; 33(1). https://doi.org/10.1186/S12302-021-00540-9

20. Ninomiya M, Ueno Y, Shimosegawa T (2014) Application of deep sequence technology in hepatology. Hepatology research: the official journal of the Japan Society of Hepatology 44(2):141- 
148. https://doi.org/10.1111/hepr.12214

21. Moradi S, Sharifi-Zarchi A, Ahmadi A, Mollamohammadi S, Stubenvoll A, Günther S, Salekdeh GH, Asgari S, Braun T, Baharvand H (2017) Small RNA Sequencing Reveals Dlk1-Dio3 Locus-Embedded MicroRNAs as Major Drivers of Ground-State Pluripotency. Stem cell reports 9(6):2081-2096. https://doi.org/10.1016/j.stemcr.2017.10.009

22. Gu X, Jin B, Qi Z, Yin X (2021) Identification of potential microRNAs and KEGG pathways in denervation muscle atrophy based on meta-analysis. Sci Rep 11(1):13560. https://doi.org/10.1038/s41598-021-92489-1

23. Zhang Y, Zhang D, Lv J, Wang S, Zhang Q (2018) miR-410-3p promotes prostate cancer progression via regulating PTEN/AKT/mTOR signaling pathway. Biochem Biophys Res Commun 503(4):24592465. https://doi.org/10.1016/j.bbrc.2018.06.176

24. Wang Y, Hou L, Yuan X, Xu N, Zhao S, Yang L, Zhang N (2020) LncRNA NEAT1 Targets FibroblastLike Synoviocytes in Rheumatoid Arthritis via the miR-410-3p/YY1 Axis. Frontiers in immunology, 11, 1975. https://doi.org/10.3389/fimmu.2020.01975

25. Huang C, Han W, Wang C, Liu Y, Chen Y, Duan Z (2019) Early Prognostic Utility of Gp210 AntibodyPositive Rate in Primary Biliary Cholangitis: A Meta-Analysis. Disease markers, 2019, 9121207. https://doi.org/10.1155/2019/9121207

26. Yoshioka Y, Taniai M, Hashimoto E, Haruta I, Shiratori K (2014) Clinical profile of primary biliary cirrhosis with features of autoimmune hepatitis: Importance of corticosteroid therapy. Hepatology research: the official journal of the Japan Society of Hepatology 44(9):947-955. https://doi.org/10.1111/hepr.12210

27. Wang J, Ye H, Zhang D, Cheng K, Hu Y, Yu X, Lu L, Hu J, Zuo C, Qian B et al (2017) Cancer-derived Circulating MicroRNAs Promote Tumor Angiogenesis by Entering Dendritic Cells to Degrade Highly Complementary MicroRNAs. Theranostics 7(6):1407-1421. https://doi.org/10.7150/thno.18262

28. Dong L, Pu Y, Zhang L, Qi Q, Xu L, Li W, Wei C, Wang X, Zhou S, Zhu J et al (2018) Human umbilical cord mesenchymal stem cell-derived extracellular vesicles promote lung adenocarcinoma growth by transferring miR-410. Cell Death Dis 9(2):218. https://doi.org/10.1038/s41419-018-0323-5

29. Liu D, Zhang N, Zhang J, Zhao H, Wang X (2016) miR-410 suppresses the expression of interleukin-6 as well as renal fibrosis in the pathogenesis of lupus nephritis. Clin Exp Pharmacol Physiol 43(6):616-625. https://doi.org/10.1111/1440-1681.12576

30. Wang H, Feng L, Zheng Y, Li W, Liu L, Xie S, Zhou Y, Chen C, Cheng D (2020) LINC00680 Promotes the Progression of Non-Small Cell Lung Cancer and Functions as a Sponge of miR-410-3p to Enhance HMGB1 Expression. OncoTargets and therapy 13:8183-8196. https://doi.org/10.2147/OTT.S259232

31. Ma ZH, Shi PD, Wan BS (2021) MiR-410-3p activates the NF-KB pathway by targeting ZCCHC10 to promote migration, invasion and EMT of colorectal cancer. Cytokine 140:155433. https://doi.org/10.1016/j.cyto.2021.155433

32. Liu X, Yao W, Xiong H, Li Q, Li Y (2020) LncRNA NEAT1 accelerates breast cancer progression through regulating miR-410-3p/ CCND1 axis. Cancer Biomark A 29(2):277-290. 
https://doi.org/10.3233/CBM-190721

33. Liu B, Zhang X, Zhang FC, Zong JB, Zhang W, Zhao Y (2013) Aberrant TGF- $\beta 1$ signaling contributes to the development of primary biliary cirrhosis in murine model. World J Gastroenterol 19(35):58285836. https://doi.org/10.3748/wjg.v19.i35.5828

34. Bogdanos DP, Gao B, Gershwin ME (2013) Liver immunology. Comprehensive Physiology 3(2):567598. https://doi.org/10.1002/cphy.c120011

35. Jadideslam G, Ansarin K, Sakhinia E, Alipour S, Pouremamali F, Khabbazi A (2018) The MicroRNA326: Autoimmune diseases, diagnostic biomarker, and therapeutic target. J Cell Physiol 233(12):9209-9222. https://doi.org/10.1002/jcp.26949

36. Xia Y, Tao JH, Fang X, Xiang N, Dai XJ, Jin L, Li XM, Wang YP, Li XP (2018) MicroRNA-326 Upregulates B Cell Activity and Autoantibody Production in Lupus Disease of MRL/Ipr Mice. Molecular therapy Nucleic acids 11:284-291. https://doi.org/10.1016/j.omtn.2018.02.010

37. Kim CJ, Lee CG, Jung JY, Ghosh A, Hasan SN, Hwang SM, Kang H, Lee C, Kim GC, Rudra D et al (2018) The Transcription Factor Ets1 Suppresses T Follicular Helper Type 2 Cell Differentiation to Halt the Onset of Systemic Lupus Erythematosus. Immunity 49(6):1034-1048e8. https://doi.org/10.1016/j.immuni.2018.10.012

38. Li L, Ma X, Zhao YF, Zhang C (2020) MiR-1-3p facilitates Th17 differentiation associating with multiple sclerosis via targeting ETS1. Eur Rev Med Pharmacol Sci 24(12):6881-6892. https://doi.org/10.26355/eurrev_202006_21678

\section{Tables}

Table 1 Clinical and laboratory characteristics of patients with PBC and CHB and healthy controls. 


\begin{tabular}{|llll|}
\hline Characteristics & PBC $(\mathrm{n}=82)$ & Control $(\mathrm{n}=20)$ & $\mathrm{CHB}(\mathrm{n}=20)$ \\
\hline Gender $(\mathrm{F} / \mathrm{M})$ & $76 / 6$ & $17 / 3$ & $15 / 5$ \\
\hline Age $($ years $\rrbracket$ & $57.2 \pm 11.0$ & $52.6 \pm 14.5$ & $55.0 \pm 6.5$ \\
\hline ALT $(\mathrm{U} / \mathrm{L})$ & $29.5(18.8 \sim 45.3)$ & $15.0(12.3 \sim 22.8)$ & $21.0(16.5 \sim 23.0)$ \\
\hline AST $(\mathrm{U} / \mathrm{L})$ & $31.5(21.0 \sim 40.3)$ & $20.0(17.0 \sim 24.8)$ & $24.0(17.3 \sim 48.0)$ \\
\hline ALP $(\mathrm{U} / \mathrm{L})$ & $160.0(118.0 \sim 242.3)$ & $59.0(49.8 \sim 73.5)$ & $65.5(53.0 \sim 91.8)$ \\
\hline GGT $(\mathrm{U} / \mathrm{L})$ & $89.9(45.5 \sim 225.4)$ & $15.5(12.0 \sim 23.3)$ & $15.0(11.0 \sim 19.0)$ \\
\hline TBil $(\mu \mathrm{mol} / \mathrm{L} \rrbracket$ & $20.4(15.0 \sim 36.2)$ & $13.0(9.3 \sim 17.0)$ & $15.3(12.4 \sim 23.6)$ \\
\hline DBil $(\mu \mathrm{mol} / \mathrm{L} \rrbracket$ & $8.2(6.6 \sim 11.6)$ & $3.0(2.0 \sim 5.4)$ & $5.6(4.0 \sim 8.2)$ \\
\hline ALB $(\mathrm{g} / \mathrm{L})$ & $35.3(32.1 \sim 37.7)$ & $46.3(44.6 \sim 47.6)$ & $44.5(37.0 \sim 47.1)$ \\
\hline GLO $(\mathrm{g} / \mathrm{L})$ & $30.3(26.9 \sim 33.3)$ & $26.9(24.1 \sim 29.1)$ & $27.6(25.3 \sim 31.0)$ \\
\hline Platelet $\left(1 \times 10^{9} / \mathrm{L}\right)$ & $165.8 \pm 70.1$ & $244.9 \pm 48.2$ & $117.7 \pm 65.8$ \\
\hline PT-INR & $1.0(0.9 \sim 1.1)$ & NA & $1.1(0.9 \sim 1.2)$ \\
\hline
\end{tabular}

Abbreviations: ALT, alanine aminotransferase; AST, aspartate transaminase; ALP, alkaline phosphatase; GGT, gamma-glutamyl-transpeptidase; TBil, total bilirubin; DBil, direct bilirubin; ALB, albumin; GLO, globulin; PT-INR, prothrombin time -international normalized ratio; NA, not available; PBC, primary biliary cholangitis ; Control, healthy controls; $\mathrm{CHB}$, chronic hepatitis B.

Table 2 Clinical and laboratory characteristics of patients with anti-gp210 positive and anti-gp210 negative PBC. 


\begin{tabular}{|c|c|c|c|}
\hline Feature & gp210-pos. $(n=36)$ & gp210-neg. $(n=46)$ & $P$ value \\
\hline Gender (F/M) & $34 / 2$ & $42 / 4$ & NS \\
\hline Age $\Downarrow$ years $\rrbracket$ & $58.6 \pm 11.8$ & $56.1 \pm 10.4$ & NS \\
\hline ALT (U/L) & $34.5(20.3 \sim 48.0)$ & $26.0(16.8 \sim 39.3)$ & NS \\
\hline AST (U/L) & $35.0(21.0 \sim 53.3)$ & $27.5(21.8 \sim 37.3)$ & NS \\
\hline ALP (U/L) & $192.0(131.6 \sim 363.6)$ & $152.0(115.6 \sim 197.0)$ & 0.014 \\
\hline GGT (U/L) & $225.0(44.6 \sim 361.0)$ & $68.0(35.0 \sim 138.0)$ & 0.004 \\
\hline TBil $(\mu \mathrm{mol} / \mathrm{LD}$ & $25.6(19.0 \sim 37.8)$ & $17.2(14.0 \sim 30.8)$ & 0.013 \\
\hline DBil $(\mu \mathrm{mol} / \mathrm{L} \rrbracket$ & $9.4(6.8 \sim 13.4)$ & $7.6(6.6 \sim 10.2)$ & NS \\
\hline ALB (g/L) & $32.3 \pm 7.0$ & $35.1 \pm 4.5$ & 0.040 \\
\hline GLO (g/L) & $31.2 \pm 7.0$ & $29.4 \pm 4.6$ & NS \\
\hline Platelet $\left(1 \times 10^{9} / \mathrm{L}\right)$ & $166.7 \pm 69.8$ & $165.1 \pm 71.1$ & NS \\
\hline PT-INR & $1.0(0.9 \sim 1.2 \rrbracket$ & $1.0(0.9 \sim 1.0 \rrbracket$ & NS \\
\hline Splenomegaly+ $(\mathrm{n}, \% 区$ & $14(38.9 \%)$ & $16(34.8 \%)$ & NS \\
\hline $\mathrm{AMA}+(\mathrm{n}, \% \mathrm{D}$ & $32(88.9 \% \square$ & $35(76.1 \%)$ & NS \\
\hline Anti-sp100 +\n, \%邓 & $8(22.2 \%)$ & $12(26.1 \%)$ & NS \\
\hline Anti-centromere + $(\mathrm{n}, \%)$ & $7(19.4 \%)$ & $19(41.3 \%)$ & 0.035 \\
\hline $\lg G(U / L)$ & $13.3(11.9 \sim 16.1)$ & $12.8(11.0 \sim 14.9)$ & NS \\
\hline $\lg M(U / L)$ & $2.3(1.2 \sim 3.2)$ & $1.8(1.1 \sim 2.5)$ & NS \\
\hline Cirrhosis + $\llbracket n, \% \bigotimes$ & $20(55.6 \%)$ & $22(47.8 \%)$ & NS \\
\hline Liver biopsy (n, \%) & $10(27.8 \%)$ & $10(21.7 \%)$ & \\
\hline Stage 1/2/3/4 (n) & $3 / 2 / 4 / 1$ & $3 / 6 / 0 / 1$ & \\
\hline Child grading $A / B / C(n)$ & $30 / 4 / 2$ & $38 / 8 / 0$ & NS \\
\hline
\end{tabular}

Abbreviations: ALT, alanine aminotransferase; AST, aspartate transaminase; ALP, alkaline phosphatase; GGT, gamma-glutamyl-transpeptidase; TBil, total bilirubin; DBil, direct bilirubin; ALB, albumin; GLO, globulin; PT-INR, prothrombin time -international normalized ratio; AMA, anti-mitochondrial antibody; IgG, immunoglobulin G; IgM, immunoglobulin M; NS, no significance; gp210-pos., anti-gp210 positive; gp210neg., anti-gp210 negative.

Table 3 Top 10 significant GO categories for miR-410-3p target genes. 


\begin{tabular}{|llll|}
\hline Term ID & Term & Count & P value \\
\hline GO:0007154 & cell communication & 164 & $8.21625 \mathrm{E}-24$ \\
\hline GO:0031325 & positive regulation of cellular metabolic process & 120 & $4.62682 \mathrm{E}-22$ \\
\hline GO:0009966 & regulation of signal transduction & 108 & $1.67543 \mathrm{E}-19$ \\
\hline GO:0031328 & positive regulation of cellular biosynthetic process & 74 & $8.80006 \mathrm{E}-15$ \\
\hline GO:0031324 & negative regulation of cellular metabolic process & 85 & $5.77692 \mathrm{E}-12$ \\
\hline GO:0034097 & response to cytokine & 66 & $2.4424 \mathrm{E}-11$ \\
\hline GO:0042981 & regulation of apoptotic process & 49 & $6.1741 \mathrm{E}-08$ \\
\hline GO:0045619 & regulation of lymphocyte differentiation & 13 & $1.17384 \mathrm{E}-06$ \\
\hline GO:0045596 & negative regulation of cell differentiation & 26 & $2.29093 \mathrm{E}-06$ \\
\hline GO:0008285 & negative regulation of cell population proliferation & 27 & $1.21975 \mathrm{E}-05$ \\
\hline
\end{tabular}

Abbreviations: GO, gene ontology ; Term ID, stands for the ID of gene ontology term; Term, stand for the name of gene ontology term; Count, stands for the number of different expression genes associated with the term.

Table 4 KEGG pathway analysis based on potential target genes of miR-410-3p.

\begin{tabular}{|llll|}
\hline Pathway ID & Name & Count & P value \\
\hline hsa04350 & TGF-beta signaling pathway & 16 & $3.46435 \mathrm{E}-06$ \\
\hline hsa04010 & MAPK signaling pathway & 29 & $4.76338 \mathrm{E}-05$ \\
\hline hsa04218 & Cellular senescence & 18 & 0.000197036 \\
\hline hsa04310 & Wnt signaling pathway & 17 & 0.001171319 \\
\hline hsa04658 & Th1 and Th2 cell differentiation & 11 & 0.002553398 \\
\hline hsa04660 & T cell receptor signaling pathway & 10 & 0.017594218 \\
\hline hsa04014 & Ras signaling pathway & 11 & 0.026764517 \\
\hline hsa04151 & Pl3K-Akt signaling pathway & 15 & 0.027608849 \\
\hline hsa04659 & Th17 cell differentiation & 9 & 0.049650716 \\
\hline
\end{tabular}

Abbreviations: KEGG, the Kyoto Encyclopedia of Genes and Genomes pathway; Pathway ID, stands for pathway identifier used in KEGG; Name, stands for the name of the pathway; Count, stands for the count of the different expression genes directly associated with the listed pathway. 


\section{Figures}

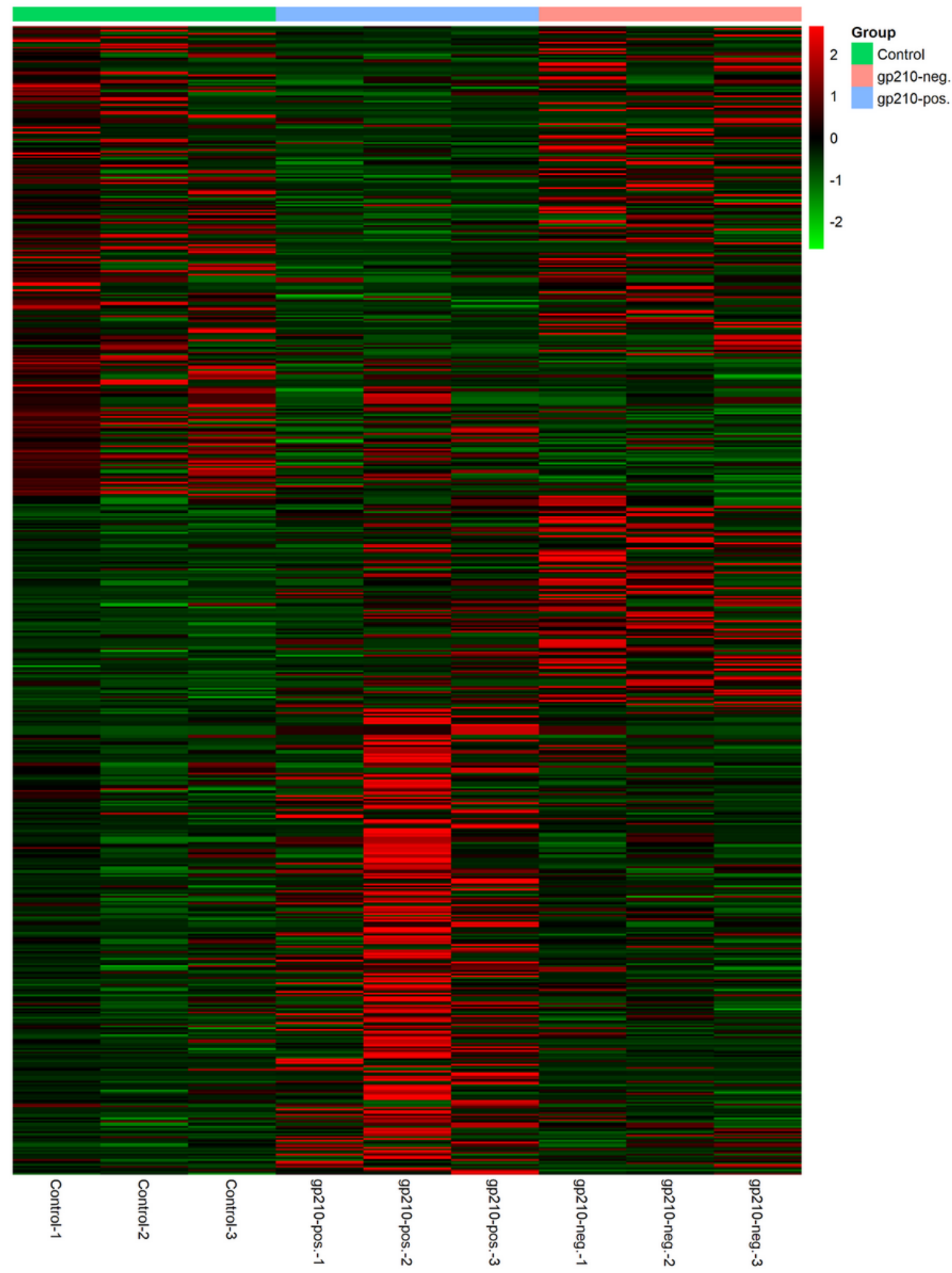

\section{Figure 1}

Heat mapping and clustering analysis. Heat mapping was performed and described using a function of heatmap. 2 in gplots. It uses hierarchical clustering with Euclidean distance. Red indicates a high level of miRNA expression and green indicates low expression. 
a

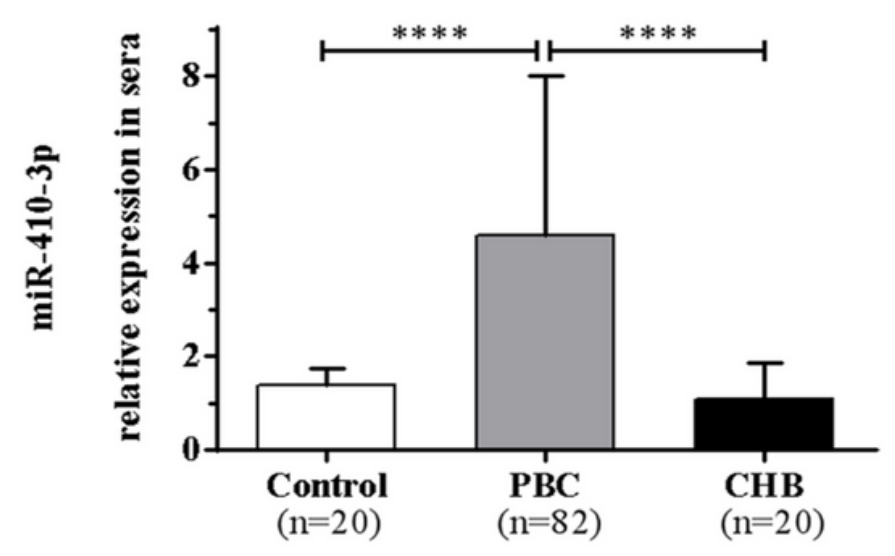

C

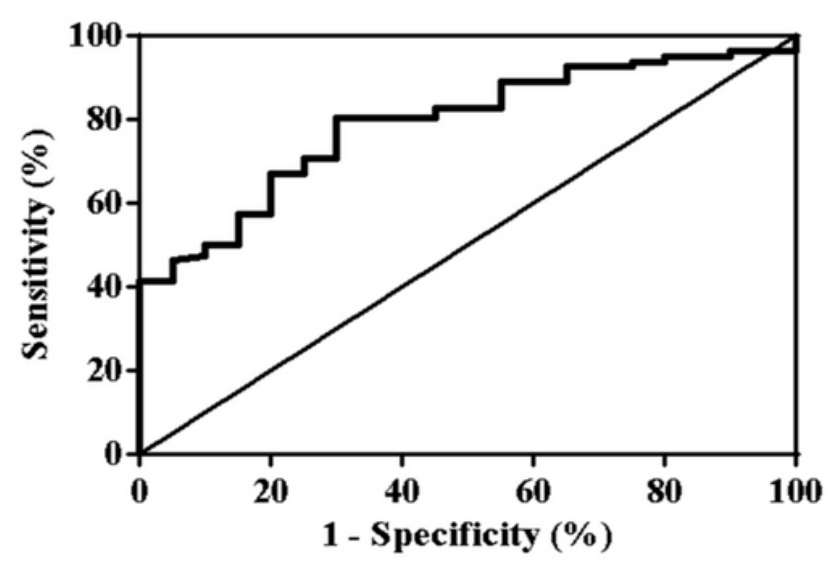

b

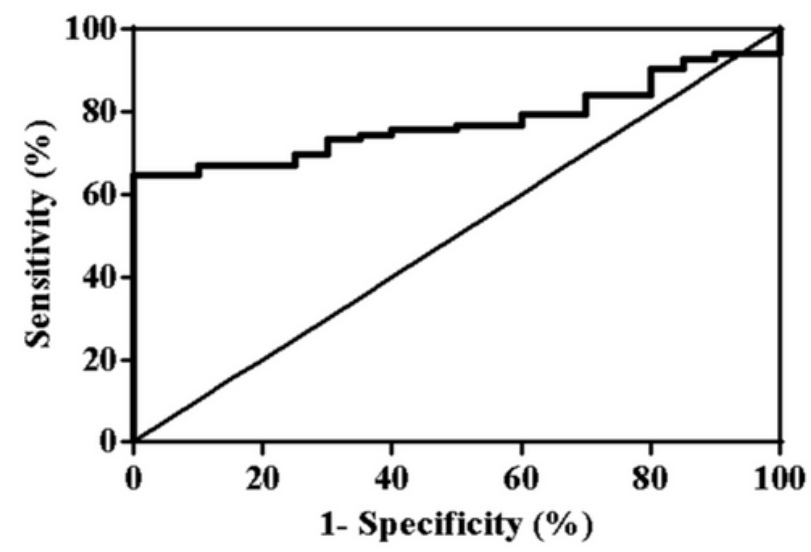

\section{Figure 2}

MiR-410-3p is specifically up-regulated in PBC patients. The expression of miR-410-3p was significantly up-regulated in PBC compared with healthy controls and CHB patients (a). ROC analysis revealed that serum miR-410-3p yielded AUC values of $0.775((95 \% \mathrm{Cl}, 0.688 \sim 0.862 ; \mathrm{p}<0.0001)$ in discriminating PBC from healthy controls (b). Serum miR-410-3p yielded AUC values of 0.791 (95\% Cl, 0.695 0.887; $\mathrm{p}<0.0001$ ) in distinguishing PBC from CHB patients (c). Control, healthy controls; PBC, primary biliary cholangitis; $\mathrm{CHB}$, chronic hepatitis $\mathrm{B}$; ROC, receiver operating characteristic; AUC, area under the curve. $\star \star \star * * p<0.0001$. 


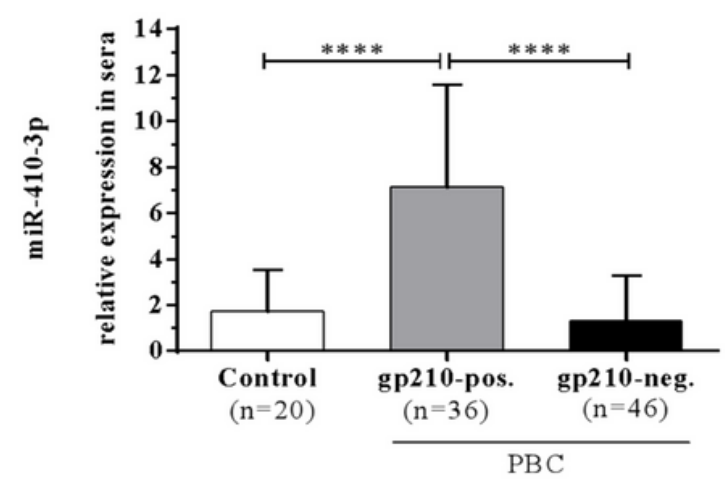

c

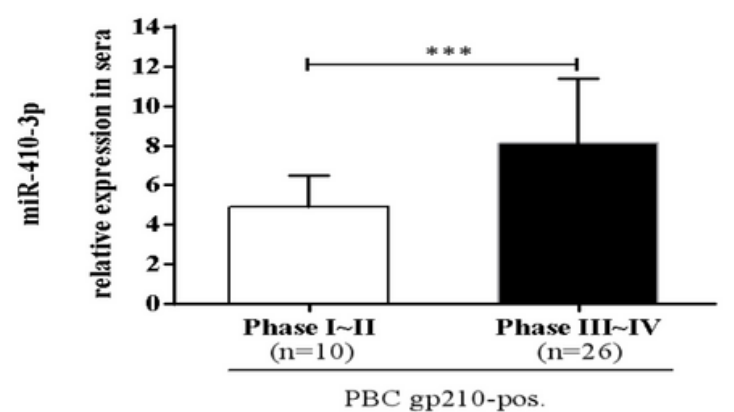

b

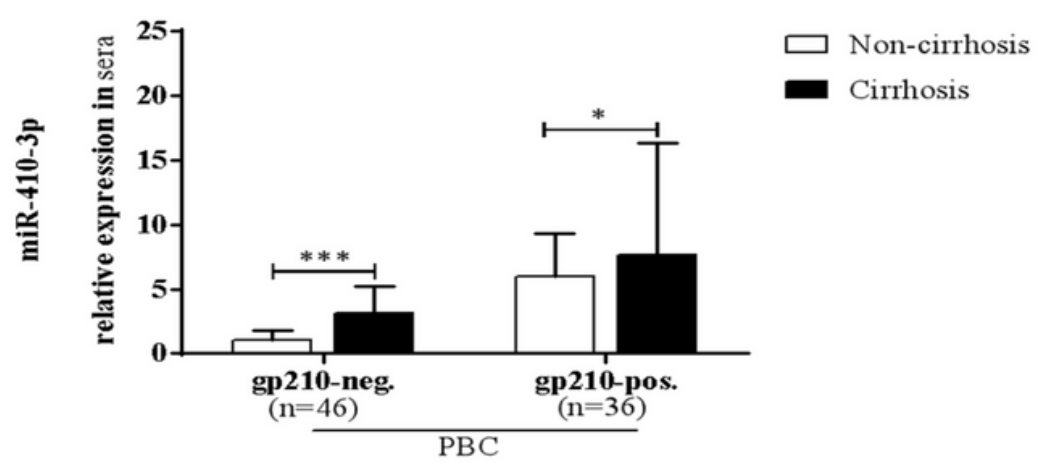

d

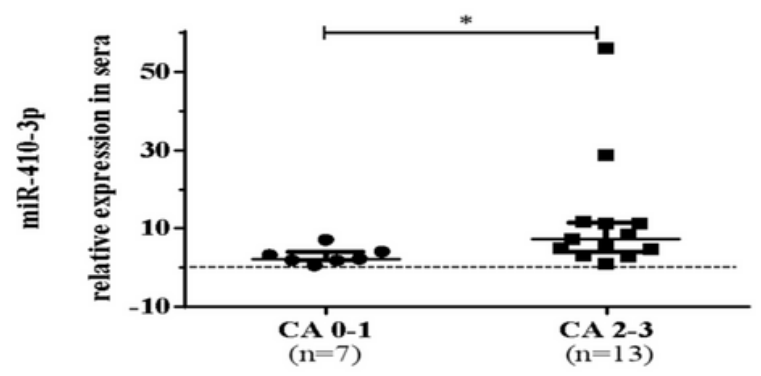

Figure 3

The relationships between glycoprotein 210 (gp210) status, the occurrence of cirrhosis and the stage of nature course, cholangitis activity (CA), response to UDCA and serum level of miR-410-3p. MiR-410-3p level was significantly higher in patients with anti-gp210 positive (gp210-pos.) PBC than in patients with anti-gp210 negative (gp210-neg.) PBC (a). The higher expression of miR-410-3p was observed in PBC patients with cirrhosis in contrast to non-cirrhotic patients (b). In patients with anti-gp210 positive PBC, miR-410-3p had a higher level in patients with nature course phase III-IV compared to patients with nature course phase I-II (c). The miR-410-3p expression level was significantly higher in PBC patients with a CA of 2-3 than in those with a CA of 0-1 (d). ${ }^{*} p<0.05,{ }^{* *} p<0.01,{ }^{* * *} p<0.001,{ }^{* * *} p<0.0001$. 


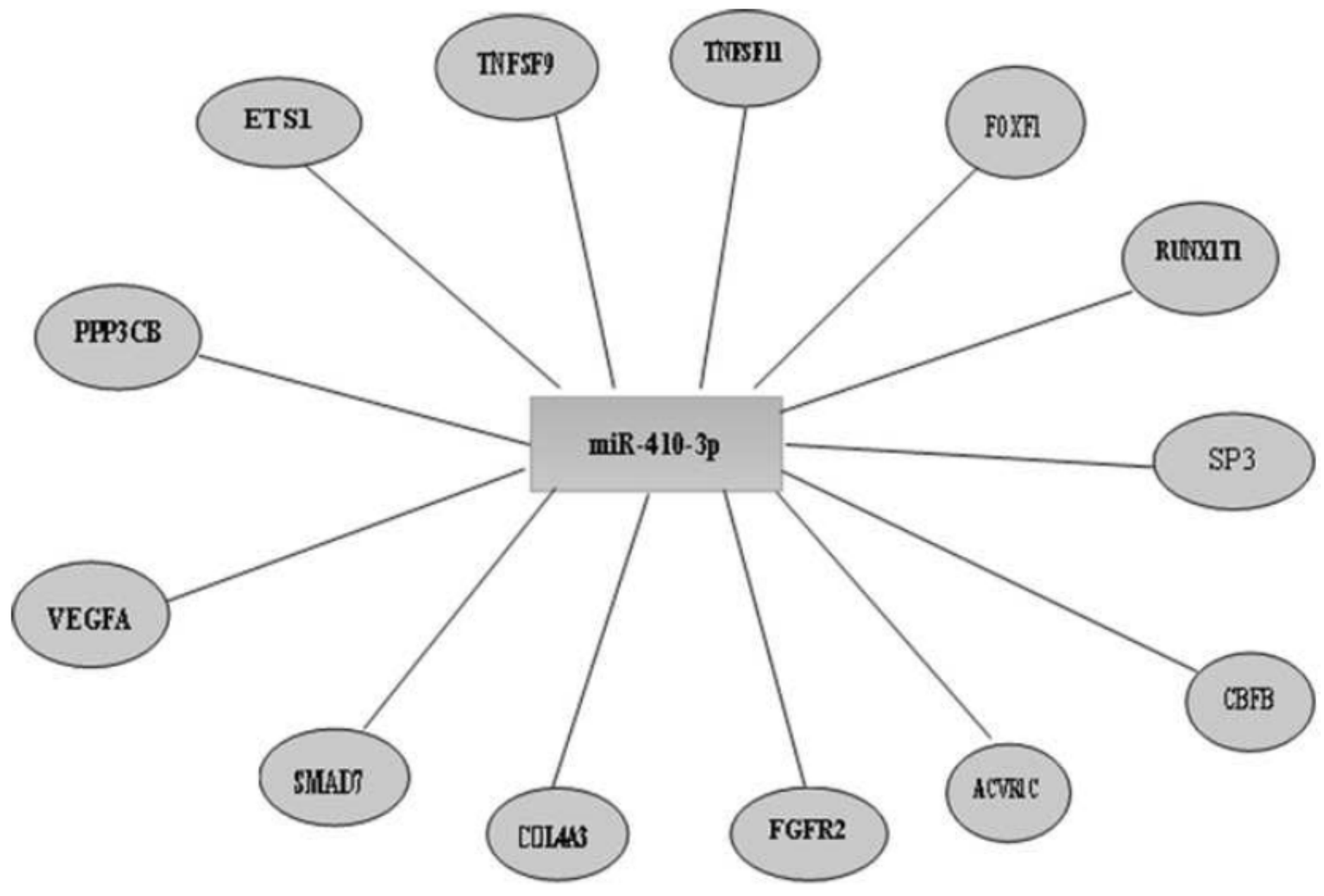

Figure 4

MicroRNA-target genes network of miR-410-3p. Target genes are related to functions of $\mathrm{T}$ cell or B cell and the occurrence and development of liver fibrosis. Gray square in the middle represents miR-410-3p; the gray ellipse on the edge represents target genes (mRNAs). The relationship between miR-410-3p and genes is represented by black line. 